\title{
Oral health of older people admitted to hospital for needs assessment
}

\author{
Ling GY, Love RM, MacFadyen EE, Thomson WM
}

\begin{abstract}
Objectives: To describe the clinical oral health status, treatment needs and oral-health-related quality of life (OHRQoL) of older people admitted to older persons' wards at Dunedin Public Hospital due to a sudden worsening of their general health.
\end{abstract}

Participants and methods: A systematic oral assessment was undertaken for a consecutive case series of 200 patients (59.5\% female; mean age 82.6 years, sd 6.6) admitted to older person's wards at Dunedin Public Hospital. The Oral Health Impact Profile-20 (OHIP-20) was used to assess OHRQOL.

Results: One in three (36.0\%) had been living independently at home prior to admission, and over half (55.0\%) had been admitted for a medical reason which required assessment. Half (50.0\%) of the participants were dentate (with an average of 16.8 teeth). There was an average of 1.9 decayed teeth present in the dentate group; $70.7 \%$ of individuals required restorations or extractions, and about $90 \%$ required only simple scaling of the teeth and prophylaxis. A reline or a replacement denture were required by three-quarters of those with dentures. Almost two-thirds of participants did not have a regular dentist, and fewer than one in three had made a dental visit in the previous year. One in six described their oral health as 'fair' or 'poor', and just under one-third reported dry mouth. Dentate participants, those without xerostomia, and those reporting better oral health had better OHRQoL, reflected in lower OHIP-20 scores. Affecting 37.1\% of participants, functional limitation was the most commonly experienced of the OHIP-20 domains, followed by physical disability and physical pain (18.0\% and $15.6 \%$ respectively).

Conclusions: The oral health of medically compromised and functionally dependent but cognitively competent older people in this study is generally poor. If transfer to long-term care is indicated, early and proper preventive measures and appropriate dental contact should be advocated in order to reduce morbidity and improve quality of life for older people.

\section{INTRODUCTION}

Most older people prefer to stay in their homes for as long as possible, rather than moving into institutional care (Thorson, 2000). Around $90 \%$ of New Zealanders aged 65 years and older live in private dwellings, over half with a spouse. However, about $7 \%$ of older people are frail and vulnerable, and require high levels of care and disability support; most (85\%) of those live in residential care facilities for older people, while about $4 \%$ are in public or private hospitals (Statistics New Zealand, 2007). In 2008, there were approximately 28,000 people in aged residential care in New Zealand. The majority reside in the more than 750 aged residential care facilities (Office for Senior Citizens, 2008) around the country. At least half of the older population in New Zealand will end up in an aged residential care facility at some stage, and only a small proportion of those will return to their own homes (Broad et al, 2013). Not only are the absolute numbers of people in care increasing, but their dependency levels have been increasing over the last two decades (Boyd et al, 2011).

Moving into residential care is usually considered when older people start to experience life changes such as deteriorating health, illness, an inability to cope at home or the loss of a caregiver. Such a move involves facing important decisions which can be overwhelming for the family and difficult for the individual. The process usually commences with a "Needs Assessment" (Ministry of Health, 2007), which identifies the required level of support, with need categorised as 'low', 'medium', 'high', or 'very high'. For older people with 'low' to 'medium' needs, the assessor works with them to develop a support "package" to help them stay at home, delaying premature admission to residential care. This can include a mix of publicly-funded and self-paid services, along with help from family and friends. If it is not possible for the older person to stay safely at home, the assessor may authorise entry to one of the residential care options. Long-term residential care is considered only for the 'high' and 'very high' categories. All New Zealand aged residential care facilities are certified by the Ministry of Health; they can be categorised as into rest homes, dementia units, or continuing care hospitals. All residential care is 24-hour institutional care provided by a mixture of nurses and paid caregivers (nurse aides, health-care assistants). Older people who are frail and in need of extra support are first assessed for the amount and type of support they need. If they are in hospital or acutely ill, a multidisciplinary teamcomprising a geriatrician, nurse, physiotherapist, occupational therapist and social worker-will make the assessment. If the individual is at home when the assessment is needed, his/ her general medical practitioner makes a referral to an agency which undertakes it on behalf of the local District Health Boards (Ministry of Health, 2007). Older people who are most likely to enter residential care are those with problems with mobility, continence or cognition, together with the very old (Weatherall et al., 2004). The InterRAI geriatric assessment tool (Centers for Medicare and Medicaid Services, 2002) is now used to provide consistency and accuracy in needs assessment.

As with those of other industrialised countries, the population of New Zealand is ageing rapidly, due to falling fertility and rising mortality, and the 65+ age group (currently $13 \%$ of the population) is predicted to comprise $23 \%$ of New 
Zealanders by 2051 (Statistics New Zealand, 2012). Occurring alongside what has been termed a "dental transition", manifested in the falling prevalence of edentulism among older people as the edentulous "oldest old" die out and the incoming "youngest old" retire with at least some of their own teeth remaining (Thomson, 2012). Currently, more than twothirds of the 65-74 group and over half of those aged 75 or more have at least some remaining natural teeth (Ministry of Health, 2010). The scarce available data on dentition status in rest homes reflect the ongoing changes: the one in five dentate residents observed in Manawatu-Horowhenua by Thomson et al (1991) was one in three in a later study by Carter et al (2004) in Christchurch. There are more older people, with more teeth, and they are known to experience high rates of dental caries, periodontitis and incremental tooth loss (Muller et al., 2007; Steele et al., 2004; Thomson, 2004; Susin et al., 2005; Thomson, 2014); those in the nursing home sector have a caries increment which is twice that of their community-dwelling counterparts (Chalmers et al, 2005). Smith (2010) highlighted complexity of the issue of oral health in the residential care sector, and stressed the need for adequate and appropriate oral health assessment of those entering care. When older people are admitted to hospital because of a health-related event, oral health can be overlooked, especially if the general health prognosis is poor and transfer to long-term care is inevitable. Little is currently known of the oral health of those who are on the threshold of entering such care, although the findings from a national survey of oral health in New Zealand rest homes (Smith et al, in press) will be released in the near future.

The aim of this study was to describe the clinical oral health status and oral-health-related quality of life (OHRQoL) of people admitted to older persons' wards at Dunedin Public Hospital due to a sudden worsening of their general health.

\section{METHODS}

Ethical approval was obtained from the Lower Southern Regional Ethics Committee. The data were obtained from a consecutive case series of older people who were admitted to Wards 6A and 6B of Dunedin Hospital from March to December 2010. Admission to Wards $6 \mathrm{~A}$ and $6 \mathrm{~B}$ is usually for assessment and rehabilitation of older people who have experienced falls, strokes, mobility problems, chronic medical conditions or social issues. The proposed sample size was 200 participants, determined with reference to a previous Dunedin study (Brown et al., 1987), and taking into account the likely level of non-participation. Data collection took place over a nine-month period. Participants were personally approached by the examiner (GYL) and informed about the study. Assessment was undertaken only when the participant agreed to take part and written informed consent had been obtained. Participants had to be aged 65 years or older. The survey included a thorough bedside clinical oral examination, an interview with each participant, and a review of that person's inpatient medical notes. For those with cognitive impairment, clinical oral examinations were carried out, but the interview was excluded (with consent obtained from the patient's next of kin or enduring power of attorney).

The inpatient medical notes of each participant were reviewed and details of social-demographic data, existing medical conditions (including the reason for admission) and current medication(s) were recorded. Routinely-recorded MiniMental Status Examination (MMSE) scores were also obtained from the inpatient medical notes; these allowed categorisation of participants according to their cognitive status (Mungas, 1991). Those scoring 26 to 30 were considered to be within the normal cognitive range; those scoring 21 to 25 had mild cognitive impairment; a score of 11 to 20 indicated moderate cognitive impairment, and those scoring 10 or less had severe cognitive impairment.

The oral examination used standard World Health Organization (WHO) methods and criteria (World Health Organization, 1997). GYL carried out all of the examinations after first having been calibrated. The oral examination used a headlight, conventional dental mirror, dental explorer and WHO standard Community Periodontal Index of Treatment Needs (CPITN) probe (the latter was used on the index teeth $17,16,11,26,27,37,36,31,46$ and 47 , with the worst score per sextant recorded). Gauze was used when necessary to keep a dry oral field, and no suction was utilised. Participants were examined in the hospital wards, either reclining on their beds or sitting in a chair. Data were collected verbally with the use of a recorder and then transferred onto the data capture form.

A questionnaire sought sociodemographic information (community services cardholder status, highest level of education, main income source and current/previous occupation). The participants' socio-economic status (SES) was determined from the latter using the Elley-Irving (males) and Irving-Elley (females) indices (Elley and Irving, 1985; Irving and Elley, 1977). Information was also sought on dental attendance and perceived need for care, along with data on oral self-care practices. Self-reported oral health data were collected using a global assessment and gobal transition judgment. The Oral Health Impact Profile-20 (OHIP-20) (Allen and Locker, 2002) was used to assess participants' oral-healthrelated quality of life (OHRQOL). The items in the OHIP capture data on seven conceptually formulated domains that are based on Locker's theoretical model of oral health (Locker, 1988): functional limitation, physical pain, psychological discomfort, physical disability, psychological disability, social disability, and handicap.

Data analysis was conducted using SPSS version 19. After the computation of univariate descriptive and summary statistics, analysis of variance and Chi-square tests (as appropriate) were used to test for the statistical significance of observed intergroup differences $(\mathrm{P}<0.05)$.

\section{RESULTS}

Sociodemographic and health characteristics

The examiner personally approached 263 patients on Wards $6 \mathrm{~A}$ and $6 \mathrm{~B}$ at Dunedin Hospital, and the first 200 patients who consented to participate were examined (giving a participation rate of $76.0 \%)$. Of those, 105 (52.5\%) were patients of Ward 6A, and $95(47.5 \%)$ were patients of Ward 6B. The participants' mean age was 82.6 years (SD, 6.6; range 65 to 98 years; median 84 ); 130 $(65.0 \%)$ were 80 years old or over. There were 81 (40.5\%) males and 119 (59.5\%) females, with the latter being just over one year older, on average. Most of the participants were NZ Europeans (92.5\%), although there were small numbers of Māori, Chinese, and Indian participants $(1.0 \%, 1.0 \%$ and $0.5 \%$ respectively). Ethnicity data were not obtained from seven participants (3.5\%). Data on the SES of participants are presented by sex and age group in Table 1 . There were significantly more participants aged 65-79 years in the medium- and low-SES groups.

Most participants (92.7\%) had lived in their own home prior to hospital admission. Of those, 72 (37.3\%) had been 
living independently, and the remainder had received assistance at home. That assistance ranged from simple house cleaning to more comprehensive support, with showering and toothbrushing included in the tasks requiring assistance. Some 14 participants $(7.3 \%)$ had been living in a rest home prior to hospital admission. Pre-admission living condition data were not obtained from seven participants. Just over half $(52.8 \%)$ of those who were living independently prior to admission were expected to be discharged home with assistance arranged, while seven (9.7\%) expected to need rest-home-level care upon discharge. Most of those who had been living with assistance (either at home or at a rest home) prior to admission expected to be discharged to the same living arrangement $(89.6 \%$ and $84.6 \%$ respectively).

Participants had been admitted for assessment and rehabilitation for various reasons, with just over half admitted for a medical reason (Table 2). The remainder had been admitted following a fall/collapse, for rehabilitation following surgery, or for other reasons. Nine participants had been admitted for reasons of cognitive deterioration, and required assessment by a geriatrician. All participants had pre-existing medical comorbidities upon admission (Table 3). These covered a wide range of pathology. The mean number of pre-existing medical conditions was 7.5 (SD, 2.7; range 2 to 17) per person. A higher proportion of those who had been living with assistance at home prior to their hospital admission had been admitted after a fall or collapse than those who were living independently (26.6\% and $6.9 \%$ respectively; $\mathrm{P}<0.05)$. The latter also had significantly fewer pre-existing medical conditions upon admission than those who had been assisted at home (6.5 and 8.0 respectively; $\mathrm{P}<0.05)$. The 200 participants were taking a mean of 10.5 different medications (SD, 4.2; range 2 to 26 ) during their hospital stay; almost three quarters (74.0\%) were taking some form of analgesic, but antihypertensives were the most common medication taken $(82.0 \%)$. Those who had been living at home independently prior to their hospital admission took significantly fewer medications than those who had been living with assistance (a mean 9.5 and 11.1 respectively; $\mathrm{P}<0.05$ ), but there were no other socio-demographic differences.

\section{Clinical oral status}

One hundred participants $(50.0 \%)$ were dentate; of those, $44(44.0 \%)$ were male and $56(56.0 \%)$ female. One dentate participant refused to undergo the dental examination, and so the dentition status data are limited to the 99 who did. One participant's dentition comprised only two teeth, and two participants had 30 teeth. Fewer than 10 teeth were present in $23.2 \%$ of the dentate participants. There were no significant differences in dentate status by age, sex or living condition prior to admission (Table 4), but a higher proportion of lowSES participants (than those of high or medium SES) were edentulous. The mean number of teeth present in the dentate group was 16.8 (SD, 7.3) per person. There were no significant differences in the mean number of teeth by socio-demographic characteristics, except that high-SES participants had more teeth (on average) than those of medium or low SES.

Forty eight $(48.5 \%)$ dentate participants had no untreated coronal caries $(\mathrm{DT}=0)$, while two-thirds $(66.6 \%)$ of the remaining 51 dentate participants had one or two DT. Data on the accumulated dental caries experience of the dentate sample
Table 1. SES group by sex and age (brackets contain percentages)

\begin{tabular}{l|rr|c|} 
& \multicolumn{3}{|c|}{ SES group } \\
& High & Medium & Low \\
\hline Sex & & & \\
$\quad$ Female & $19(18.4)$ & $60(58.3)$ & $24(23.3)$ \\
$\quad$ Male & $18(23.7)$ & $42(55.3)$ & $16(21.1)$ \\
Age group & & & \\
$\quad 65-79$ & $8(12.7)$ & $44(69.8)$ & $11(17.5)^{\mathrm{a}}$ \\
$\quad 80+$ & $29(25.0)$ & $58(50.0)$ & $29(25.0)$ \\
All combined & $37(20.7)$ & $102(57.0)$ & $40(22.3)$ \\
& & &
\end{tabular}

a $<0.05$

Table 2. Reasons for hospital admission

\begin{tabular}{|c|c|}
\hline Reason for admission & $\begin{array}{l}\text { Number of } \\
\text { participants (\%) }\end{array}$ \\
\hline \multicolumn{2}{|l|}{ Medical: } \\
\hline Pain management & $34(17.0)$ \\
\hline Cerebrovascular accident/stroke & $23(11.5)$ \\
\hline Respiratory & $11 \quad(5.5)$ \\
\hline Diabetes & $9 \quad(4.5)$ \\
\hline Infection & $6 \quad(3.0)$ \\
\hline Urology & $6 \quad(3.0)$ \\
\hline Nervous system & $6 \quad(3.0)$ \\
\hline Generally unwell & $5 \quad(2.5)$ \\
\hline Gastrointestinal & $4 \quad(2.0)$ \\
\hline Cardiovascular & $2(1.0)$ \\
\hline Haematology & $2(1.0)$ \\
\hline Oncology & $1 \quad(0.5)$ \\
\hline Renal & $1(0.5)$ \\
\hline Subtotal & $110(55.0)$ \\
\hline \multicolumn{2}{|l|}{ Post-surgery: } \\
\hline Elective orthopaedic surgery & $11 \quad(5.5)$ \\
\hline Gastrointestinal surgery & $9 \quad(4.5)$ \\
\hline Orthopaedic surgery due to fractures & $3(1.5)$ \\
\hline Cardiovascular surgery & $3(1.5)$ \\
\hline Prostate surgery & $1 \quad(0.5)$ \\
\hline Vascular surgery & $1 \quad(0.5)$ \\
\hline Cranial surgery & $1 \quad(0.5)$ \\
\hline Subtotal & $29(14.5)$ \\
\hline \multicolumn{2}{|l|}{ Falls/Collapse: } \\
\hline Accidental & $26(13.0)$ \\
\hline Medical & $13(6.5)$ \\
\hline Total & $39(19.5)$ \\
\hline \multicolumn{2}{|l|}{ Others: } \\
\hline Confusion/Cognitive decline & $9 \quad(4.5)$ \\
\hline Decreased mobility & $7 \quad(3.5)$ \\
\hline $\begin{array}{l}\text { Unable to cope at home/social } \\
\text { /safety reasons }\end{array}$ & $6 \quad(3.0)$ \\
\hline Subtotal & $22(11.0)$ \\
\hline All combined & $200(100.0)$ \\
\hline
\end{tabular}


are presented in Table 5. On average, females had significantly more coronal and root surface restorations than males, and high-SES participants had more filled teeth, fewer missing teeth, and more sound roots.

Caries-associated treatment needs were apparent in 70 dentate participants $(70.7 \%)$, with the most common being

Table 3. Pre-existing medical conditions among participants

\begin{tabular}{|c|c|}
\hline Category of medical condition & $\begin{array}{c}\text { Number of } \\
\text { participants }(\%)^{\mathrm{a}}\end{array}$ \\
\hline Cardiovascular & $169(84.5)$ \\
\hline Connective tissues, joints and bones & $135 \quad(67.5)$ \\
\hline Nervous system & $100 \quad(50.0)$ \\
\hline Endocrine & $95 \quad(47.5)$ \\
\hline Gastrointestinal & $89 \quad(44.5)$ \\
\hline Kidney and urinary & $83(41.5)$ \\
\hline Respiratory & $55 \quad(27.5)$ \\
\hline Haematology & 39 (19.5) \\
\hline Dermatological & $33(16.5)$ \\
\hline Psychiatric & $29 \quad(14.5)$ \\
\hline Liver & $15 \quad(7.5)$ \\
\hline Infection & $13 \quad(6.5)$ \\
\hline Miscellaneous & $140 \quad(70)$ \\
\hline
\end{tabular}

aPercentages do not total $100 \%$ because each participant had more than one medical condition restorative treatment, needed by 59 (59.6\%); $23.2 \%$ required three or more restorations, and four participants each required six restorations. Of the 28 participants $(28.3 \%)$ who required extractions, most required only one. There were no systematic differences by sociodemographic characteristics or expected post-discharge functional dependency.

The CPITN data (Table 6) showed that most participants had calculus or minor pocketing, and that three individuals had deeper pockets requiring more sophisticated periodontal therapy than scaling and prophylaxis.

One-third (33.3\%) of the 99 dentate participants were functionally dentate; that is, they had an adequate anterior occlusion comprising between 16 and 20 teeth and wore no intra-oral prosthesis. Of the remaining 66 dentate participants, $53(80.3 \%)$ were partially dentate and were wearing some form of removable prosthesis. Of those, 26 (39.4\%) were wearing a full upper denture (and nine of those were opposing a lower partial denture), 25 (37.9\%) wore an upper partial denture (seven of which opposed a lower partial, and one opposed a full lower denture), and 2 (3.0\%) wore just a lower partial denture. Among the 100 edentulous participants, one wore no dentures, three wore a full upper denture but no lower denture, and the remainder wore full upper and lower dentures (with one participant's lower denture being implant-retained). Clinical assessment revealed that three-quarters of the edentulous participants would require a reline or a new set of dentures.

Use of dental services

Just over one-third (35.1\%) of participants were regular dental attenders, and 59 (31.9\%) reported having visited a dentist in the previous twelve months; among dentate and edentulous

Table 4. Dental status of participants by socio-demographic and general health characteristics (brackets contain percentages unless otherwise indicated)

\begin{tabular}{|c|c|c|c|}
\hline & \multicolumn{2}{|c|}{ Dentition status } & \multirow{2}{*}{$\begin{array}{l}\text { Mean no of teeth among } \\
\text { dentate persons (SD) }\end{array}$} \\
\hline & Edentulous & Dentate & \\
\hline \multicolumn{4}{|l|}{ Sex } \\
\hline Female & $63(52.9)$ & $56(47.1)$ & $16.9(7.0)$ \\
\hline Male & $37(45.7)$ & $44(54.3)$ & $16.7(7.8)$ \\
\hline \multicolumn{4}{|l|}{ Age group } \\
\hline $65-79$ & $32(45.7)$ & $38(54.3)$ & $18.6(7.1)$ \\
\hline $80+$ & $68(52.3)$ & $62(47.7)$ & $15.8(7.3)$ \\
\hline \multicolumn{4}{|l|}{ SES group ${ }^{a}$} \\
\hline High & $13(35.1)$ & $24(64.9)^{b}$ & $20.9(6.5)^{b}$ \\
\hline Medium & $49(48.0)$ & $53(52.0)$ & $16.1(6.8)$ \\
\hline Low & $27(67.5)$ & $13(32.5)$ & $14.4(7.7)$ \\
\hline \multicolumn{4}{|l|}{ Pre-admission domicile } \\
\hline At home, independent & $30(41.7)$ & $42(58.3)$ & $17.8(7.7)$ \\
\hline Other & $70(54.7)$ & $58(45.3)$ & $16.2(7.0)$ \\
\hline \multicolumn{4}{|c|}{ Expected functional dependency after discharge $\mathrm{e}^{\mathrm{C}}$} \\
\hline Similar & $67(50.4)$ & $66(49.6)$ & $16.6(6.9)$ \\
\hline Greater & $28(48.3)$ & $30(51.7)$ & $18.2(7.9)$ \\
\hline All combined & $100(50.0)$ & $100(50.0)$ & $16.8(7.3)$ \\
\hline
\end{tabular}

${ }^{a}$ Missing data for 21 participants

${ }^{\mathrm{b}} \mathrm{P}<0.05$

'Missing data for 9 participants 
Table 5. Summary data on dental caries experience, by sociodemographic characteristics (dentate participants only)

\begin{tabular}{|c|c|c|c|c|c|c|c|c|}
\hline & \multicolumn{4}{|c|}{ Coronal caries experience } & \multicolumn{4}{|c|}{ Root surface caries experience } \\
\hline & Mean DT & Mean MT & Mean FT & $\begin{array}{l}\text { Mean } \\
\text { DMFT }\end{array}$ & Mean DT & Mean FT & Mean DFT & $\begin{array}{l}\text { Mean } \\
\text { number } \\
\text { of sound } \\
\text { roots }\end{array}$ \\
\hline \multicolumn{9}{|l|}{ Sex } \\
\hline Female & $1.1(1.6)$ & $15.0(7.0)$ & $10.7(6.6)^{\mathrm{a}}$ & $26.7(4.2)^{\mathrm{a}}$ & $0.8(1.1)$ & $2.9(3.1)^{\mathrm{a}}$ & $3.7(3.0)^{\mathrm{a}}$ & $12.1(6.4)$ \\
\hline Male & $1.5(1.9)$ & $14.6(7.9)$ & $7.4(6.4)$ & $23.5(5.8)$ & $1.0(1.5)$ & $1.3(1.9)$ & $2.2(2.8)$ & $13.3(7.8)$ \\
\hline \multicolumn{9}{|l|}{ Age group } \\
\hline $65-79$ & $1.3(1.9)$ & $13.2(7.1)$ & $10.4(8.1)$ & $25.0(4.8)$ & $0.9(1.4)$ & $1.8(2.2)$ & $2.6(2.5)$ & $14.5(7.0)^{\mathrm{a}}$ \\
\hline $80+$ & $1.2(1.7)$ & $15.8(7.4)$ & $8.5(5.6)$ & $25.5(5.5)$ & $0.9(1.3)$ & $2.4(2.9)$ & $3.3(3.2)$ & $11.5(6.8)$ \\
\hline \multicolumn{9}{|l|}{ SES group ${ }^{b}$} \\
\hline High & $1.0(1.4)$ & $11.0(6.6)^{\mathrm{a}}$ & $12.5(5.9)^{\mathrm{a}}$ & $24.5(4.4)$ & $0.6(0.9)$ & $2.5(2.3)$ & $3.1(2.4)$ & $16.3(6.8)^{\mathrm{a}}$ \\
\hline Medium & $1.4(2.0)$ & $15.3(7.0)$ & $8.6(6.5)$ & $25.3(5.5)$ & $1.0(1.4)$ & $1.8(2.2)$ & $2.8(2.7)$ & $12.1(6.8)$ \\
\hline Low & $1.3(1.7)$ & $17.6(7.7)$ & $6.9(6.8)$ & $25.8(5.2)$ & $0.4(0.7)$ & $1.3(1.7)$ & $1.8(1.7)$ & $11.6(7.1)$ \\
\hline \multicolumn{9}{|l|}{ Preadmission domicile } \\
\hline At home, independent & $1.2(1.9)$ & $13.5(7.7)$ & $9.4(7.1)$ & $24.1(5.9)$ & $0.6(0.8)$ & $2.0(2.1)$ & $2.3(2.2)$ & $13.6(6.8)$ \\
\hline Other & $1.3(1.7)$ & $15.8(7.0)$ & $9.1(6.4)$ & $26.1(4.5)$ & $1.1(1.5)$ & $2.2(3.1)$ & $3.3(3.4)$ & $11.9(7.1)$ \\
\hline \multicolumn{9}{|l|}{$\begin{array}{l}\text { Expected functional de- } \\
\text { pendency after discharge }\end{array}$} \\
\hline Similar & $1.1(1.6)$ & $15.3(7.0)$ & $9.3(6.7)$ & $25.8(4.5)$ & $0.9(1.4)$ & $2.1(2.6)$ & $3.0(2.8)$ & $12.7(6.7)$ \\
\hline Greater & $1.7(2.1)$ & $12.8(7.8)$ & $9.2(6.8)$ & $23.7(6.4)$ & $0.7(0.9)$ & $1.9(1.9)$ & $2.6(2.2)$ & $13.6(7.4)$ \\
\hline All combined & $1.3(1.8)$ & $14.8(7.4)$ & $9.2(6.7)$ & $25.3(5.2)$ & $0.9(1.3)$ & $2.2(2.7)$ & $3.0(3.0)$ & $12.6(7.0)$ \\
\hline
\end{tabular}

${ }^{\mathrm{a}} \mathrm{P}<0.05$

${ }^{\mathrm{b}}$ Data missing for 10 participants

'Data missing for 4 participants

participants, $57.0 \%$ and $6.5 \%$ respectively $(\mathrm{P}<0.05)$ had visited in that period. Of the 126 who reported not having done so, more than two-thirds (69.7\%) felt that they did not have any dental problems; other reasons included being too ill (3.2\%), cost $(2.4 \%)$, too difficult to get to the dentist $(2.4 \%)$, not called in $(1.6 \%)$, no time to go $(1.6 \%)$, and two participants mentioned that they were either afraid of the dentist or not able to cope with dental treatment.

Just over one-third of participants (36.0\%) felt that they currently needed some form of dental treatment. Among those who expected to be discharged into a living situation with a greater level of assistance, that proportion was nearly half $(47.3 \%)$.

Self-reported oral health and oral-health-related quality of life (OHRQoL)

Self-reported oral health information was obtained from 178 participants, of whom $46.1 \%$ reported that their oral health in general was 'Good', while one in six (16.8\%) reported it to be 'Fair' to 'Poor', and $6.2 \%$ and $30.9 \%$ rated it as 'Excellent' or 'Very good', respectively. When asked to rate changes in their oral health over the previous three months, most $(83.6 \%)$ reported no change, $10.7 \%$ believed that their oral health had deteriorated, and $5.6 \%$ had improved.

Complete OHIP-20 data were available for 162 participants $(81.0 \%)$, whose mean total OHIP-20 score was 11.9 (SD, 10.5; range 0 to 50). There were no socio-demographic differences in OHIP-20 scores, but edentulous participants had a higher mean total OHIP-20 score than those who were dentate (14.7 and 9.1 respectively; $\mathrm{P}<0.01)$. One or more OHIP-20 impacts (fairly/very often) was experienced by $43.7 \%$ of participants; that was higher among those aged 65-79 than it was among those aged 80 or older $(50.0 \%$ and $29.2 \%$ respectively; $\mathrm{P}<0.05)$. Those experiencing one or more OHIP-20 impacts had higher mean root DT $(0.6$ and 1.5 respectively; $\mathrm{P}<0.05)$.

\section{DISCUSSION}

This study set out to describe the clinical oral health status and self-reported oral health of a consecutive case series of people admitted to older persons' wards at Dunedin Public Hospital due to a sudden worsening of their general health. It has found that they had substantial unmet dental treatment needs but were not a homogeneous group; there was a range of functional dependency and domiciliary states prior to admission. Half of the sample was edentulous. Among the dentate participants, caries-associated treatment needs predominated, and onequarter required an extraction.

Before discussing the findings, it is appropriate to first consider the weaknesses and strengths of the study. The principal weakness is that it used a consecutive clinical sample rather than a representative one. This was dictated by logistical concerns and resource constraints; the study was undertaken as a doctoral research project, and the candidate (GYL) needed to be able to collect the data locally. This meant that the numbers used in the study were dictated by the patient flow at Dunedin Public Hospital. Moreover, there is no other feasible way of sampling those who are being admitted for 
Table 6. Summary data on the Community Periodontal Index of Treatment Needs (CPITN)

\begin{tabular}{|c|c|c|c|c|c|}
\hline \multicolumn{6}{|c|}{ A. The numbers and percentage of participants who had, as the highest score: } \\
\hline & Healthy & Bleeding & Calculus & $4-5 \mathrm{~mm}$ pocket & $6+\mathrm{mm}$ pocket \\
\hline Score & 0 & 1 & 2 & 3 & 4 \\
\hline Number & 5 & 2 & 48 & 41 & 3 \\
\hline Percentage & 5.1 & 2.0 & 48.5 & 41.4 & 3.0 \\
\hline \multicolumn{6}{|c|}{ B. The numbers and percentage of participants needing: } \\
\hline & No treatment & $\begin{array}{l}\text { Improved oral } \\
\text { hygiene }\end{array}$ & Scaling & \multicolumn{2}{|c|}{ Complex therapy } \\
\hline Number & 5 & 2 & 89 & \multicolumn{2}{|c|}{3} \\
\hline Percentage & 5.1 & 2.0 & 89.9 & \multicolumn{2}{|c|}{3.0} \\
\hline
\end{tabular}

assessment: a representative sample could be obtained from those already residing in care or from people who are likely to require it at some stage (such as those who remain in their own homes with assistance funded by the Ministry of Health), but neither of those would have provided people undergoing the transition from one to the other. Another weakness is that the periodontal data were not collected in detail (with only the CPI and index teeth used), but the challenges of examining this particular group of older people in far-from-ideal examination circumstances mean that this was the only option available. The study's principal strenght is the breadth of the data collected, with not only clinical examinations, but also self-report data, including OHRQoL and recent use of dental services.

Turning to the findings, the older people included in the study are best described as "elderly", medically compromised and functionally dependent, but cognitively competent. The data show that the oral health of these older people is poor, and there is a definite need for oral health care. The participants' low perceived needs and lack of dental contact raise considerable concerns. As a group of older people who are transitioning through a major deterioration of their general health, and becoming increasingly frail and dependent, it is often a very stressful time for them and their families. With deteriorating general health, oral health may often be forgotten, and it is not surprising that older people in these circumstances will place their oral health needs on hold while concentrating on more pressing concerns. Their oral health problems will tend to accumulate if left unchecked, and the older person is left with both poor oral and general health. Furthermore, delivering oral health care to these older people can present additional challenge, not only to the dental profession, but also to the rest home sector (Antoun et al., 2008; Smith, 2010).

Preventing oral disease before they become frail appears to be a valid strategy for older people. As people live longer and retain more natural teeth, the complexity of their associated treatment needs will only increase. Oral health care for older people should be realistic and "future proofed", anticipating changes in health. From the workforce perspective, a wider team approach within the oral health profession is essential. While dentists would be the primary oral health care provider, dental auxiliary personnel (dental hygienists and/or therapists) should act as oral health coordinators and provide oral hygiene treatment, some conservative treatment, and training for relevant staff within institutions (Smith, 2010). Although the use of a team approach in providing oral health care to older people is still in its infancy in New Zealand (Moffat et al., 2009), the concept is not new (Chalmers, 2003; Thomson et al., 1991), and it would be invaluable in many respects, especially given that the wider dental profession does not presently have the capacity or the incentive to become involved (Antoun et al, 2008).

It is also equally important that oral health prevention strategies with older people should extend beyond the traditional walls of dental practices. Since older people tend to visit their doctor far more frequently than they visit dentists (Cautley et al., 1992; Dolan and Atchison, 1993), oral health promotion strategies should include those other health professionals. General medical practitioners would be in the ideal position to point out the importance for (and impact of) good oral health on the general well-being of older people. Given the findings of the current study, one of the important oral health strategies would be to include an oral health assessment as part of the overall health assessment during hospital admission for older people, as urged by Smith (2010) and more latterly by Pretty et al (2014). A baseline oral assessment should focus on dentate status, oral hygiene status and ability, and the presence of dental caries or oral pathology. Moreover, it is important to ask about whether they are experiencing any oral symptoms. If an oral symptom is evident, appropriate dental contact should be advocated in a timely manner to ensure good oral health is achieved for older people before they become frail. The routine use of an OHRQoL scale would provide useful baseline data against which subsequent changes in oral health could be monitored.

It is important to acknowledge that oral health is integral to general health, and a determinant of quality of life (Petersen and Yamamoto, 2005; World Health Organization, 2006). Oral health promotion strategies at all levels should better integrate oral health and general health needs of older people, their carers and other health professionals. If transfer to long-term care is indicated, early and proper preventive measures and appropriate dental contact should be advocated in order to reduce morbidity and improve quality of life for older people.

\section{ACKNOWLEDGEMENTS}

We thank the study participants, as well as Professor AJ Campbell (University of Otago) for his advice. We are grateful to the Ministy of Health for funding the study. 


\section{REFERENCES}

Allen F, Locker D (2002). A modified short version of the oral health impact profile for assessing health-related quality of life in edentulous adults. Int J Prosthodont 15: 446-450.

Antoun JS, Adsett LA, Goldsmith SM, Thomson WM (2008). The oral health of older people: general dental practitioners' beliefs and treatment experience. Spec Care Dent 28: 2-7.

Boyd M, Broad JB, Kerse N, Foster S, von Randow M, Lay-Yee R, Chelimo C, Whitehead N, Connolly MJ (2011). Twenty-year trends in dependency in residential aged care in Auckland, New Zealand: a descriptive study. Journal of the American Medical Directors Association 120: 535-540.

Broad JB, Gott M, Kim H, Boyd M, Chen H, Connolly MJ (2013). Where do people die? An international comparison of the percentage of deaths occurring in hospital and residential aged care settings in 45 populations, using published and available statistics. Int J Public Health 58: 257-267.

Brown RH, Edwards JL, Spears GF (1987). Periodontal and tooth treatment needs in dentate elderly people in Dunedin. NZ Dent J 83: 42-5.

Carter G, Lee M, McKelvey V, Sourial A, Halliwell R, Livingston $M$ (2004). Oral health status and oral treatment needs of dependent elderly people in Christchurch. NZ Med J 117: U892.

Cautley AJ, Rodda JC, Treasure ET, Spears GF (1992). The oral health and attitudes to dental treatment of a dentate elderly population in Mosgiel, Dunedin. NZ Dent J 88: 138-43.

Chalmers JM (2003). Oral health promotion for our ageing Australian population. Aust Dent J 48: 2-9.

Chalmers JM, Carter KD, Spencer AJ (2005). Caries incidence and increments in Adelaide nursing home residents. Spec Care Dent 25: 96-105.

Dolan TA, Atchison KA (1993). Implications of access, utilization and need for oral health care by the non-institutionalized and institutionalized elderly on the dental delivery system. $J$ Dent Educ 57: 876-887.

Elley W, Irving J (1985). The Elley-Irving Socio-Economic Index 1981 Census Revision. NZ J Educ Stud 20: 115-128.

Irving J, Elley W (1977). A Socio-Economic Index for the Female Labour Force in New Zealand. NZ J Educ Stud 12: 154-163.

Ministry of Health (2007). Looking at Long-term Residential Care in a Rest Home or Hospital: What you need to know: Wellington: Ministry of Health.

Ministry of Health (2010). Our oral health. Key findings of the 2009 New Zealand Oral Health Survey. Wellington: Ministry of Health.

Moffat SM, Coates DE, Meldrum AM (2009). New Zealand's changing oral health workforce. A dental practitioner's guide to dual-trained dental therapists/dental hygienists. NZ Dent J 105: 57-61.

Muller F, Naharro M, Carlsson GE (2007). What are the prevalence and incidence of tooth loss in the adult and elderly population in Europe? Clinical Oral Implants Research 18: 2-14.

Mungas D (1991). In-office mental status testing: a practical guide. Geriatrics 46: 54-58.

New Zealand Dental Association (2010). Healthy Mouth, Healthy Ageing: Oral Health Guide for Caregivers of Older People. Auckland: New Zealand Dental Association.
Office for Senior Citizens (2008). Briefing to the incoming minister - New Zealanders: getting older, doing more: http:// www.msd.govt.nz/documents/about-msd-and-our-work/ publications-resources/corporate/bims/osc-bim-2008.pdf, last accessed: 20 June 2011.

Petersen PE (2003). The World Oral Health Report 2003: continuous improvement of oral health in the 21st century the approach of the WHO Global Oral Health Programme. Community Dentistry \& Oral Epidemiology 31: 3-23.

Petersen PE, Yamamoto T (2005). Improving the oral health of older people: the approach of the WHO Global Oral Health Programme. Community Dentistry \& Oral Epidemiology 33: 81-92.

Pretty IA, Ellwood RP, Lo ECM, MacEntee MI, Müller F, Rooney ER, Thomson WM, Van der Putten G, Ghezzi EM, Walls AWG (2014). The Seattle Care Pathway for securing oral health in older patients. Gerodontol 31 (Suppl 1): 77-87.

Smith MB (2010). Oral health and well-being of older adults in residential aged-care facilities: issues for public health policy. NZ Dent J 106: 67-73.

Smith MB, Thomson WM, Gribben B. 2012 Older people's oral health survey. Key findings from the study into older people's oral health issues. Wellington: Ministry of Health. (In press)

Statistics New Zealand (2007). New Zealand's 65+ Population: A Statistical volume. Wellington: Statistics New Zealand.

Statistics New Zealand. Demographic Trends: 2011. Wellington: Statistics New Zealand, 2012.

Steele JG, Sanders AE, Slade GD, Allen PF, Lahti S, Nuttall N, et al. (2004). How do age and tooth loss affect oral health impacts and quality of life? A study comparing two national samples. Community Dent Oral Epidemiol 32: 107-114.

Susin C, Oppermann RV, Haugejorden O, Albandar JM (2005). Tooth loss and associated risk indicators in an adult urban population from south Brazil. Acta Odont Scand 63: 85-93.

Thomson WM, Brown RH, Williams SM (1991). Dental status of a New Zealand institutionalised elderly population. NZ Dent J 87: 119-123 (1991).

Thomson WM (2004). Dental caries experience in older people over time: what can the large cohort studies tell us? Brit Dent J 196: 89-92.

Thomson WM (2012). Monitoring edentulism in older New Zealand adults over two decades: a review and commentary. Int $J$ Dent Volume 2012, Article ID 375407, doi:10.1155/2012/375407.

Thomson WM (2014). Epidemiology of oral health conditions in older people. Gerodontol 31 (Suppl 1): 9-16.

Thorson J (2000). Aging in a changing society. 2nd Edition ed. New York: Brunner-Routhledge.

Weatherall M, Slow T, Wiltshire K (2004). Risk factors for entry into residential care after a support-needs assessment. NZ Med J117: U1075.

World Health Organization (1997). Oral Health Surveys Basic Methods. 4th Edition ed. Geneva: World Health Organization

World Health Organization (2006). Oral Health in Ageing Societies. Integration of oral health and general health. Geneva: World Health Organization. 